OPEN ACCESS

Edited by: Yin Fang,

Nanyang Technological University,

Singapore

Reviewed by: Bin Li,

Soochow University, China Marian H. Hettiaratchi, University of Oregon, United States

*Correspondence:

Zuhao $\mathrm{Li}$ lizuhao1992@163.com Minfei Wu wuminfei100@163.com

Jincheng Wang

wangjinc@jlu.edu.cn

${ }^{\dagger}$ These authors have contributed equally to this work

Specialty section: This article was submitted to Biomaterials,

a section of the journal Frontiers in Bioengineering and Biotechnology

Received: 06 August 2021 Accepted: 12 October 2021 Published: 04 November 2021

Citation:

Wang X, Li Z, Wang Z, Liu H, Cui Y, Liu Y, Ren M, Zhan H, Li Z, Wu M and Wang J (2021) Incorporation of Bone

Morphogenetic Protein-2 and Osteoprotegerin in 3D-Printed Ti6Al4V Scaffolds Enhances Osseointegration Under Osteoporotic Conditions. Front. Bioeng. Biotechnol. 9:754205. doi: 10.3389/fbioe.2021.754205

\section{Incorporation of Bone Morphogenetic Protein-2 and Osteoprotegerin in 3D-Printed Ti6Al4V Scaffolds Enhances Osseointegration Under Osteoporotic Conditions}

\author{
Xianggang Wang ${ }^{1,2 \dagger}$, Zhengyan $\mathrm{Li}^{3,4 t}$, Zhonghan Wang ${ }^{1,2}$, He Liu ${ }^{1,2}$, Yutao Cui ${ }^{1,2}$, \\ Yuzhe Liu ${ }^{1,2}$, Ming Ren ${ }^{1,2}$, Hongsheng Zhan ${ }^{3,4}$, Zuhao $\mathrm{Li}^{1,2 *}$, Minfei Wu ${ }^{1,2 *}$ and \\ Jincheng Wang ${ }^{1,2 *}$ \\ ${ }^{1}$ Orthopaedic Medical Center, The Second Hospital of Jilin University, Changchun, China, ${ }^{2}$ Orthopaedic Research Institute of Jilin \\ Province, Changchun, China, ${ }^{3}$ Shi's Center of Orthopedics and Traumatology, Shuguang Hospital Affiliated to Shanghai \\ University of TCM, Shanghai, China, ${ }^{4}$ Institute of Traumatology, Shanghai Academy of TCM, Shanghai, China
}

Osteoporosis is an age-related metabolic disease that results in limited bone regeneration capacity and excessive osteoclast activity. After arthroplasty in patients with osteoporosis, poor interface osseointegration resulting from insufficient bone regeneration ability often leads to catastrophic complications such as prosthesis displacement and loosening and periprosthetic fractures. In this study, we prepared a thermosensitive hydrogel loaded with bone morphogenetic protein-2 (BMP-2) to promote osteogenesis and osteoprotegerin (OPG) to inhibit excessive osteoclast activity. To construct three-dimensional (3D)-printed composite scaffolds for implantation, a hydrogel loaded with drugs was injected into porous Ti6Al4V scaffolds. The 3D-printed composite scaffolds showed good biocompatibility and sustained release of BMP-2 and OPG for more than 20 days. In vitro experiments indicated that composite scaffolds promoted osteogenic differentiation and reduced the osteoclastic activation simultaneously. Remarkably, immunofluorescence staining, micro-CT, histological, and biomechanical tests demonstrated that the sustained release of both BMP-2 and OPG from composite scaffolds significantly improved bone ingrowth and osseointegration in osteoporotic defects. In conclusion, this study demonstrated that the BMP-2- and OPG-loaded 3D-printed composite scaffolds can potentially promote osseointegration for osteoporotic patients after joint replacement.

Keywords: osteoporosis, bone tissue engineering, bone morphogenetic protein-2, osteoprotegerin, osseointegration

\section{INTRODUCTION}

Osteoporosis is one of the most common metabolic skeletal diseases, and it is characterized by low bone strength, limited osteogenic activity, and enhanced osteoclast resorption, thus increasing the risk of bone fractures (Ferrari, 2018; Compston et al., 2019). In bone tissue, there is a dynamic metabolic process of bone modeling and remodeling. Osteoblasts mediate the modeling process via secreting bone matrix and promoting calcium deposition. However, osteoclasts mediate the remodeling process via bone matrix resorption (Guo et al., 2021; Jia et al., 2021). However, the 
balance between bone formation and bone resorption in osteoporotic patients is disrupted, thus resulting in poor osseointegration after prosthesis implantation (Toepfer et al., 2021). So far, various conventional treatments have been attempted to increase the osseointegration of osteoporosis patients after joint replacement, but these treatments have not alleviated the problem (Monotti et al., 2020; Quinzi et al., 2020). Postoperative systemic administration of antiresorptive drugs is limited by various side effects and low bioavailability that consistently achieves an unsatisfactory local osseointegration effect (Frank et al., 2021). Poor osseointegration after joint replacement leads to serious complications, including prosthesis loosening or displacement and periprosthetic fracture (Zhu et al., 2021). Therefore, to ameliorate the pathological environment in the osteoporotic interface, an effective treatment that can promote osseointegration at the prosthetic interface after joint replacement is urgently required.

The titanium alloy, Ti6Al4V, is an excellent material for fabricating orthopedic implants because of its good biocompatibility, osteoconductivity, and superior corrosion resistance (Huang et al., 2021). However, the high stiffness of scaffolds causes stress shielding and poor interfacial bonding, which remains a significant problem (Abate et al., 2021). To address this challenge, $3 \mathrm{D}$ printing technology has been used to fabricate prostheses with a highly porous structure (Li et al., 2021). The controlled pore structure not only reduces the difference in stiffness between the prosthesis and host bone but also provides enough space for bioactive substance loading and induces bone ingrowth. Bone morphogenetic proteins (BMPs) are classic growth factors, which can induce osteogenic differentiation of stem cells in vitro and bone regeneration in vivo (Baek et al., 2021). Among all the BMPs, BMP-2 is one of the most osteogenic BMPs that can significantly promote bone induction. In the clinic, recombinant human BMP2 (rhBMP-2) is already approved by the Food and Drug Administration (FDA) for human use (Chao et al., 2021). BMP-2 can strongly induce bone formation via the SMAD signaling pathway in osteoblasts (Fitzpatrick et al., 2017). In the beginning, BMP-2 will bind to the BMP receptor on the cell membrane, and then it will initiate a cascade of BMP-specific SMAD pathways, which eventually promote the expression of osteogenesis-related genes (Wang et al., 2020). In previous studies, BMP-2 was used for bone regeneration in osteoporotic defects and showed excellent results (Segredo-Morales et al., 2018; Garcia-Garcia et al., 2019). In addition, considering the special microenvironment of osteoporosis, it is necessary to block its excessive osteoclastic activity. Osteoprotegerin (OPG) is a glycoprotein belonging to the tumor necrosis factor (TNF) receptor superfamily, which is considered to be a factor that inhibits bone resorption. OPG is mainly expressed by osteoblasts; it functions to inhibit osteoclastogenesis and osteolysis via binding and neutralizing RANKL (Hauser et al., 2017). OPG could decrease the activity of osteoclasts by regulating the RANKL/RANK/OPG system in osteoporosis (Tu et al., 2015).

In this study, we prepare the thermosensitive poloxamer 407 hydrogels as a drug delivery system to incorporate BMP-2 and OPG, and the hydrogel loaded with drugs was injected into the pores of 3D-printed Ti6Al4V scaffolds to construct composite scaffolds. The Ti6Al4V scaffold with optimized pore size and porosity is designed to match the bone tissue's mechanical strength, thus minimizing the stress shielding. We hypothesize that these BMP-2/OPG-loaded composite scaffolds will release drugs continuously and ameliorate the regenerating microenvironment in osteoporosis. Moreover, the cooperation between BMP-2 and OPG will enhance bone regeneration and inhibit bone resorption simultaneously, thus enhancing the osseointegration after the prosthesis implantation (Scheme 1).

\section{EXPERIMENTAL SECTION}

\section{Materials}

The Ti6Al4V powder was purchased from AK Medical Co., Ltd. (Beijing, China). Poloxamer 407 powder was obtained from Bayee Chemical Co., Ltd. (Hangzhou, China). BMP-2, OPG, and antibodies used in immunofluorescence were obtained from Abcam (Cambridge, UK). Low Glucose Dulbecco's Modified Eagle's Medium (DMEM), streptomycin doubleantibody, and fetal bovine serum (FBS) were obtained from Gibco (Grand Island, NY, USA). Paraformaldehyde and phosphate-buffered saline (PBS) were obtained from Coolabar (Beijing, China). Cell Counting Kit-8 (CCK-8) and Calcein-AM/ propidium iodide (PI) were supplied by Beyotime Biotechnology (Shanghai, China). The osteogenic medium used for alizarin red dye was obtained from Cyagen (Santa Clara, CA, USA). The Perfect Real-Time RE reagent kit from Takara Bio (Dalian, China), 2× Fast SYBR Green Master Mix from Roche Diagnostics (Basel, Switzerland), and TRIzol reagent (Invitrogen, CA, USA) were used. The Runx-2, OPN, and RANKL antibodies were supplied by Abcam (Cambridge, UK) and DAPI from Solarbio (Beijing, China). The Electrochemical Immunoassay Kit was obtained from Roche-Mannheim (Mannheim, Germany). The tartrate-resistant acid phosphatase (TRAP) staining kit was obtained from Sigma-Aldrich (St. Louis, MO, USA). The ultrapure water used in the study was obtained from a Milli-QA10 filtration system (Millipore, Billerica, MA, USA).

\section{Preparation of Bone Morphogenetic Protein-2/Osteoprotegerin-Loaded 3D-Printed Composite Scaffolds}

\section{Fabrication of the 3D-Printed Ti6Al4V Scaffolds}

The 3D-printed porous Ti6Al4V scaffolds were fabricated as previously described (Bai H. et al., 2020). Briefly, a 3D model of the disk-shaped scaffold ( $\Phi 6 \mathrm{~mm} \times \mathrm{L} 3 \mathrm{~mm}$ ) was established with the following parameters: pore size $=800 \mu \mathrm{m}$, porosity $=$ $70 \%$, and strut size $=300 \mu \mathrm{m}$. Spherical pre-alloyed medicalgrade Ti6Al4V powder (Grade 23, particle size $45-100 \mu \mathrm{m}$ ) was used to fabricate the porous scaffold using the electron beam melting (EBM) machine (Q10, Arcam, Gothenburg, Sweden). To verify whether the parameters of the prepared scaffolds were consistent with the predesign, a scanning electron microscope (SEM; JSM-6700F, JEOL, Japan) and Micro-CT scanner (SkyScan 


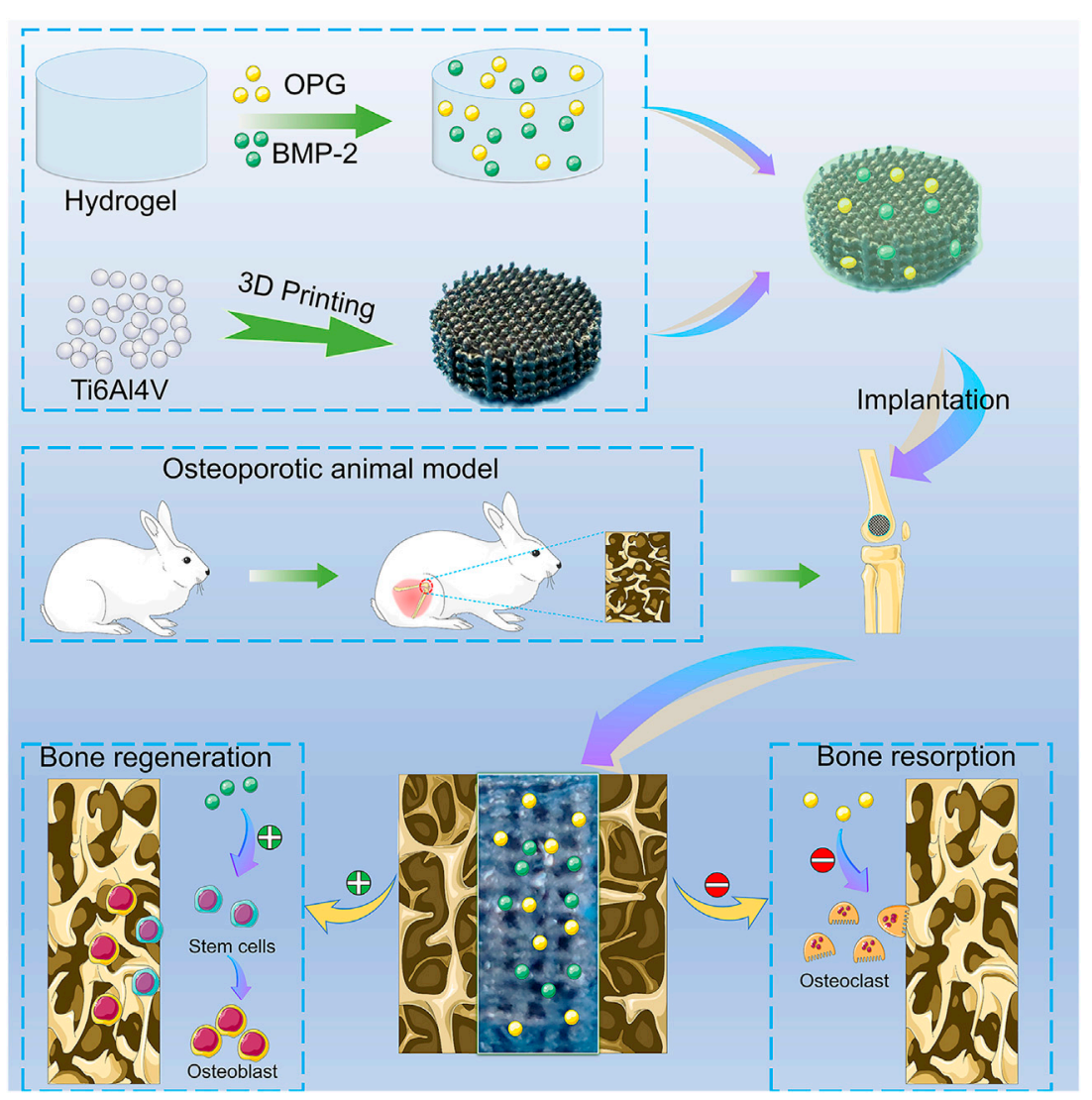

SCHEME 1 | The illustration of the BMP-2/OPG-loaded 3D-printed composite scaffolds and their application as scaffolds to promote osseointegration in osteoporosis. BMP-2, bone morphogenetic protein-2; OPG, osteoprotegerin.

1076, Kontich, Belgium) were used to evaluate the pore size and porosity. All obtained scaffolds were ultrasonically cleaned and washed sequentially in acetone, ethanol, and deionized water for $10-15 \mathrm{~min}$ in each solution.

\section{Preparation of the Hydrogel}

The hydrogel was prepared by mixing the powder with $0.01 \mathrm{M}$ of PBS $\left(\mathrm{pH}=7.4,4^{\circ} \mathrm{C}\right)$ at the ratio of $25 \%: 75 \%(\mathrm{w} / \mathrm{w})$ at $4^{\circ} \mathrm{C}$ overnight. The rheological properties of the poloxamer 407 hydrogels were assessed on a rheometer (Discovery HR-2, TA Instruments, New Castle, DE, USA) from a Peltier plate. The dynamic mechanical data of the storage modulus $\left(G^{\prime}\right)$ and viscous modulus $\left(G^{\prime \prime}\right)$ of the hydrogels were tested at different temperatures. Moreover, to observe the morphology of the hydrogel and scaffolds, the SEM was also used at $3-\mathrm{kV}$ acceleration voltage.

\section{Construction of 3D-Printed Composite Scaffolds}

After poloxamer 407 was completely dissolved, a homogeneous and transparent solution was obtained. Then, the BMP-2 solution $\left(7.5 \mu \mathrm{g} / \mathrm{ml}\right.$, in $0.01 \mathrm{M}$ of PBS, $\left.\mathrm{pH}=7.4,4^{\circ} \mathrm{C}\right)$ and the OPG solution $\left(0.1 \mathrm{mg} / \mathrm{ml}\right.$, in $0.01 \mathrm{M}$ of $\left.\mathrm{PBS}, \mathrm{pH}=7.4,4^{\circ} \mathrm{C}\right)$ were added to the prepared hydrogel solution. To prepare the drug-loaded composite scaffolds, the porous Ti6Al4V scaffolds were inserted into a 96-well plate, and the hydrogel containing BMP-2 and OPG was injected into the porous Ti6Al4V scaffolds at $4^{\circ} \mathrm{C}$. Finally, the composite scaffolds within the $96-$ well plate were warmed to $37^{\circ} \mathrm{C}$ in an incubator to gelate the hydrogel solution.

\section{Bioactive Bone Morphogenetic Protein-2 and Osteoprotegerin Release Profile}

To investigate the bioactive release of BMP-2 and OPG in vitro, the BMP-2/OPG-loaded 3D-printed composite scaffolds were put in a 24 -well culture plate. It was soaked in PBS at $37^{\circ} \mathrm{C}$. Medium measuring $2 \mathrm{ml}$ was collected and changed at predetermined time points. BMP-2 and OPG release levels were tested using the BMP-2 and OPG ELISA Kits and analyzed by a microplate reader (Multiskan EX, Thermo Fisher Scientific, Shanghai, China).

\section{In Vitro Cell Experiments Isolation and Culture of Osteoporosis-Derived Bone Marrow Mesenchymal Stem Cells}

The osteoporosis-derived bone marrow mesenchymal stem cells (OP-BMSCs) were cultured and isolated as previously described (Zhao et al., 2020). Briefly, the OP-BMSCs were obtained from the long bone marrow of female New Zealand rabbits 10 months 
after ovariectomy (OVX). OP-BMSCs were cultured in a lowglucose DMEM containing 1\% streptomycin-penicillin and 10\% FBS in a humidified incubator at $37^{\circ} \mathrm{C}$ and $5 \% \mathrm{CO}_{2}$. Cells were digested and passaged at approximately $80 \%$ confluence. The third passage of cells was used for in vitro experiments.

\section{Biocompatibility}

The CCK-8 experiment was used to investigate the cell proliferation of composite scaffolds. OP-BMSCs with a density of $1 \times 10^{4}$ cells/well in 24-well culture plates were seeded in porous scaffolds (S), hydrogel-incorporated porous scaffolds (SH), BMP-2-loaded hydrogel-incorporated porous scaffolds (SH/BMP-2), OPG-loaded hydrogel-incorporated porous scaffolds (SH/OPG), and dual BMP-2 and OPG-loaded hydrogel-incorporated porous scaffolds (SH/Dual). The cell proliferation was tested on days 1,4 , and 7 . At every time point, the reaction solution was added to each well for $2.5 \mathrm{~h}$ of incubation at $37^{\circ} \mathrm{C}$. The optical density (OD) was measured using a microplate reader at $450 \mathrm{~nm}$. Calcein-AM/PI staining was performed after 3 days of culture according to the manufacturer's protocol to determine the cell viability in each group. Briefly, $2 \mu \mathrm{M}$ of Calcein-AM and $4.5 \mu \mathrm{M}$ of PI were added to the samples, incubated for $15 \mathrm{~min}$ at $37^{\circ} \mathrm{C}$ in the dark, and then evaluated under a confocal laser scanning microscope (CLSM; FV1000, Olympus, Tokyo, Japan).

\section{Alizarin Red S Staining}

The OP-BMSCs were seeded on the S, SH, SH/BMP-2, SH/OPG, and SH/Dual groups at a density of $1 \times 10^{5}$ cells/well in a 24 -well plate. After the cells adhered, the medium was changed to an osteogenic induction medium. The medium was changed every 3 days. The osteogenic differentiation ability was evaluated at 14 and 21 days. At the scheduled time point, cells were fixed with $4 \%$ paraformaldehyde for $30 \mathrm{~min}$ at $37^{\circ} \mathrm{C}$ and washed three times with PBS. The ARS solution was added to the samples at room temperature for $20 \mathrm{~min}$. After being washed with PBS to remove the residual stains, the images were taken using a microscope (Olympus IX). To quantify the results of ARS, samples were dissolved with $10 \%$ cetylpyridinium chloride, and the coloration of each sample was read by a microplate reader at $562 \mathrm{~nm}$.

\section{Real-Time Quantitative PCR}

After osteogenic induction culture of OP-BMSCs $\left(1 \times 10^{5}\right.$ cells/ well) in 24-well plates for 14 and 21 days, the expression of runtrelated transcription factor-2 (Runx-2), osteopontin (OPN), and receptor activator of NF- $\kappa \mathrm{B}$ ligand $(R A N K L)$ were investigated using RT-qPCR. Total RNA was collected using TRIzol reagent. The purity of RNA was evaluated by a NanoDrop 2,000c Spectrophotometer (Thermo Fisher Scientific, Waltham, MA, USA). Only an $A_{260} / A_{280}$ value around 2.0 was accepted for further analysis. Synthesis of cDNA was performed in a $20-\mu l$ reaction volume via a Perfect Real-Time RE reagent kit. Then, the qPCR amplification and detection were analyzed using $2 \times$ Fast SYBR Green Master Mix on a LightCycler 480 (Bio-Rad CFX Manager 3.1, Hercules, CA, USA). Based on the gene expression ratio among different groups, the relative expression level of mRNA was normalized to GAPDH and calculated using the
$2^{-\Delta \Delta c t}$ method. Primer sequences are listed in Supplementary Table S1.

\section{In Vivo Osseointegration of 3D-Printed Composite Scaffolds Preparation of Osteoporotic Animal Models}

Animal experiments were conducted in compliance with the National Institutes of Health's Guide for the Care and Use of Laboratory Animals (NIH Publications No. 8023, revised 1978). All experimental protocols for animals were approved by the Animal Care and Use Ethics Committee at Jilin University. The osteoporotic rabbit models were prepared by OVX according to our previous studies (Bai H. et al., 2020). Briefly, a total of 46 female New Zealand rabbits were randomly divided into two groups; namely, 43 of them underwent bilateral OVX surgery, and three of them received sham surgery. Ten months later, their serum estrogen levels were tested using an Electrochemical Immunoassay Kit. Three rabbits of each group were sacrificed, and their distal femurs were used for Micro-CT measurement and $\mathrm{H} \& \mathrm{E}$ staining to confirm the osteoporosis status.

\section{Implantation of 3D-Printed Composite Scaffolds}

Forty residual osteoporotic rabbits were enrolled in the osseointegration experiments, and they were randomly divided into five groups: $\mathrm{S}, \mathrm{SH}, \mathrm{SH} / \mathrm{BMP}-2, \mathrm{SH} / \mathrm{OPG}$, and SH/Dual. Briefly, the osteoporotic rabbits were anesthetized using 3\% (w/v) pentobarbital $(50 \mathrm{mg} / \mathrm{kg})$. After the skin preparation and sterilization, a longitudinal incision at the distal femur site was made to expose the lateral condyle. Then, a cylindrical bone defect (6.0 $\mathrm{mm}$ in diameter and $3.0 \mathrm{~mm}$ in depth) was created with a bone drill. 3D-printed composite scaffolds were implanted into each animal, and incisions were closed subsequently in layers by absorbable sutures. After the operation, all rabbits were allowed free movement. Penicillin (40,000 U) was intramuscularly injected for 3 days to prevent infection postoperatively. Three months after the implantation, all rabbits were sacrificed by overdose with $3 \%$ $(\mathrm{w} / \mathrm{v})$ pentobarbital $(150 \mathrm{mg} / \mathrm{kg})$, and the bilateral femurs were harvested for subsequent detection.

\section{Micro-CT Analysis}

The specimens were scanned using Micro-CT (90 kV, 114 mA, 18$\mu \mathrm{m}$ image pixel size) to evaluate the effect of bone regeneration and osseointegration. A cylinder $(6.0-\mathrm{mm}$ diameter and $3.0-\mathrm{mm}$ height) was selected as the region of interest (ROI). The $3 \mathrm{D}$ reconstruction was performed by multimodal $3 \mathrm{D}$ visualization software (NRecon 1.7.1.0 software, Kontich, Belgium). The quantitative analysis of the ROI was conducted by Micro-CT auxiliary software (VG studio Volume Graphics $\mathrm{GmbH}$, Heidelberg, Germany), including bone volume/tissue volume ratio (BV/TV, \%), trabecular thickness (Tb.Th, $\mathrm{mm}$ ), trabecular separation (Tb.Sp, mm), and trabecular number (Tb.N, 1/mm).

\section{Immunofluorescence Staining}

As previously reported, the immunofluorescence staining of osteogenesis-related genes at the regenerated bone site was carried out (Zhao et al., 2020). Briefly, bone tissue sections were 


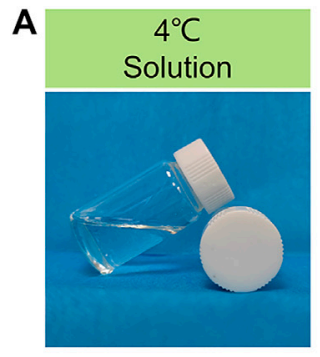

C
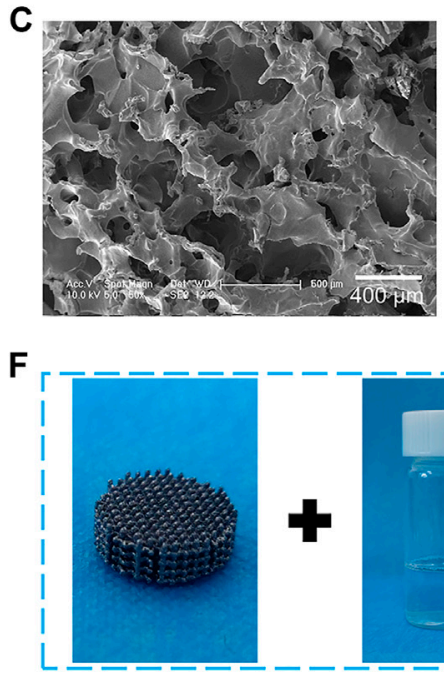

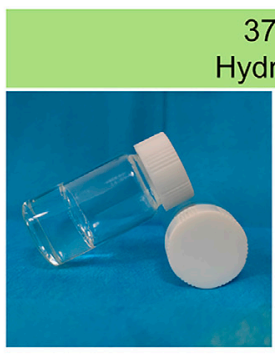

$37^{\circ} \mathrm{C}$

Hydrogel
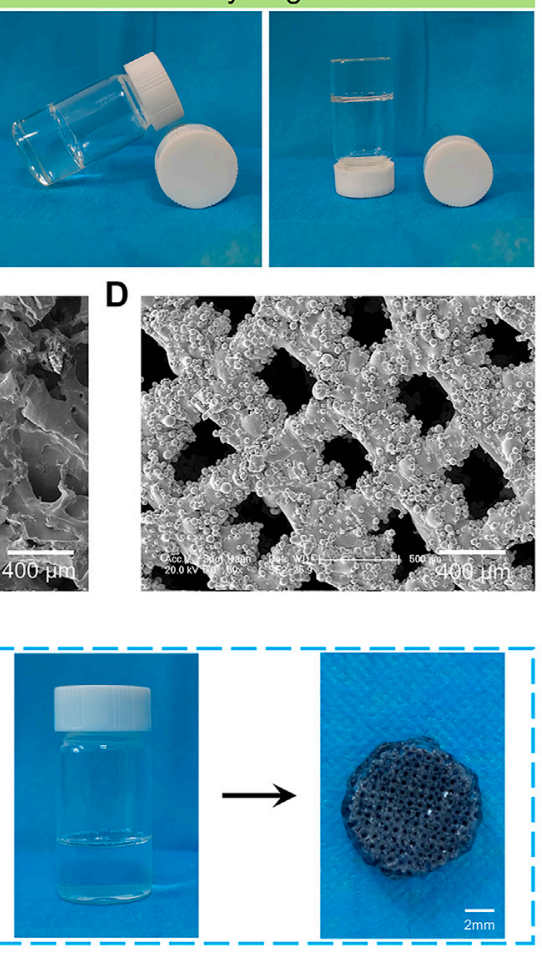

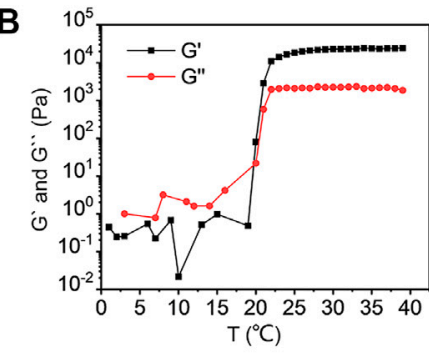

E

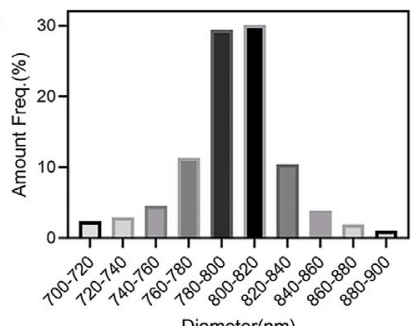

G

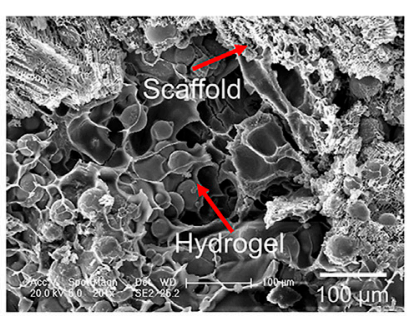

FIGURE 1 | Characterization of the BMP-2/OPG-loaded 3D-printed composite scaffolds. (A) The thermosensitive property of the poloxamer 407 hydrogel. (B) The rheological analysis of the storage modulus $\left(G^{\prime}\right)$ and loss modulus $\left(G^{\prime \prime}\right)$ of the hydrogel as a function of temperature. (C) Morphologies of the hydrogels observed by SEM. (D) Morphologies of the 3D-printed porous Ti6Al4V scaffolds. (E) The pore size distribution of 3D-printed porous Ti6Al4V scaffolds. (F) Illustration demonstrating the hydrogel loaded with drugs injected into porous scaffolds to prepare the BMP-2/OPG-loaded 3D-printed composite scaffolds. (G) SEM image of the 3D-printed composite scaffolds. BMP-2, bone morphogenetic protein-2; OPG, osteoprotegerin; SEM, scanning electron microscopy.

incubated with Runx-2, OPN, and RANKL antibodies overnight at $4^{\circ} \mathrm{C}$. After that, the specimens were washed three times using PBS, and cell nuclei were stained with DAPI. The positively stained samples were imaged with a CLSM; the intensity of fluorescence was quantitatively analyzed by image Pro-Plus (IIP) 6.0 (National Institutes of Health, Bethesda, MD, USA).

\section{Push-Out Test}

A standard push-out test was performed to evaluate the integration strength of the bone-scaffold interface in each group. The specimens were placed on the plate for a detaching test $(1.0 \mathrm{~mm} / \mathrm{min})$ using a closed-loop servo-hydraulic testing machine (MTS MiniBionix, Minneapolis, MN, USA). The maximum pushing force was recorded when scaffolds moved away from the bone.

\section{Statistical Analysis}

All data were represented as the mean \pm SD from at least three independent experiments. The statistical analyses were performed via Student's t-test or one-way ANOVA, followed by the least significant difference test for multiple comparisons using SPSS 19.0 software (SPSS Inc., Chicago, IL, USA). $p<0.05$ was deemed to indicate statistical significance.

\section{RESULTS AND DISCUSSION}

\section{Preparation and Characterization of 3D-Printed Composite Scaffolds}

The poloxamer 407 hydrogel is widely used in bone tissue engineering because of its reversible thermo-responsive properties, superior drug delivery capabilities, excellent biocompatibility, and biodegradability (Yang et al., 2020). In this study, thermosensitive poloxamer 407 hydrogels were successfully prepared (Figure 1A). The poloxamer 407 solution was liquid at $4^{\circ} \mathrm{C}$, and it was converted to a gel state when the temperature rose to $37^{\circ} \mathrm{C}$. Furthermore, a rheological analysis of the thermosensitive hydrogel confirmed that both the storage modulus $\left(G^{\prime}\right)$ and loss modulus $\left(G^{\prime \prime}\right)$ of the hydrogel rapidly increased after $21^{\circ} \mathrm{C}$, suggesting that the temperature of the hydrogel to sol-gel transition was about $21^{\circ} \mathrm{C}$ (Figure 1B). SEM observations showed that the hydrogel had a porous internal structure with a pore size of about $100-200 \mu \mathrm{m}$ (Figure 1C). The thermosensitive property of the poloxamer 407 hydrogel made it suitable for bone tissue engineering because the normal human body temperature is $37^{\circ} \mathrm{C}$.

In addition, the $3 \mathrm{D}$-printed porous Ti6Al4 $\mathrm{V}$ scaffolds were successfully fabricated using the EBM machine. SEM images of the porous scaffolds revealed the interconnected pore structure 
(Figure 1D). A quantitative analysis (using ImageJ software) showed that the diameter of micropores ranged from 760 to $840 \mu \mathrm{m}$ (Figure 1E), and the average pore size was $791.97 \pm$ $11.32 \mu \mathrm{m}$. Subsequently, the porosity of the prepared porous Ti6Al4V scaffolds was determined by Micro-CT. The results revealed that the porosity of the scaffolds was $70.13 \% \pm 2.43 \%$. Therefore, the actual pore size and porosity of the printed porous Ti6Al4V scaffolds were consistent with the predesign model ( $800 \mu \mathrm{m}$ and $70 \%$, respectively). The osseointegration ability of the porous prosthesis after implantation is closely related to the porosity and pore size. For porous implants, the porosity should be above $50 \%$, specifically in the range of $65 \%-75 \%$. Its structure and mechanics are bionic with human trabecular bone, which is conducive to osseointegration. As for the pore size, previous research has revealed that when the pore is greater than $300 \mu \mathrm{m}$, it is beneficial for nutrients and oxygen to penetrate the interconnected micropores (Chen et al., 2020). Furthermore, studies on the relationship between micropore diameter and bone regeneration have indicated that a pore size between 600 and $800 \mu \mathrm{m}$ is beneficial for osseointegration in osteoporosis (Bai $\mathrm{H}$. et al., 2020; Qiao et al., 2020). Therefore, the pore size and porosity of our prepared porous scaffolds were $791.97 \mu \mathrm{m}$ and $70.13 \%$, respectively, which are ideal for osseointegration in osteoporosis.

The synthesis procedures for BMP-2/OPG-loaded 3D-printed composite scaffolds are depicted in Figure 1F. At $4^{\circ} \mathrm{C}$, the porous Ti6Al4V scaffold was soaked in the BMP-2/OPG-loaded poloxamer 407 solutions to fill the scaffold pores uniformly. At $37^{\circ} \mathrm{C}$, the solution turned into a hydrogel, and the hydrogel and the scaffold were combined into a complex. The SEM images also confirmed that the scaffold pores were filled with hydrogels (Figure 1G). These results indicated that the BMP-2/OPG-loaded $3 \mathrm{D}$-printed composite scaffolds were prepared successfully.

Hydrogels are always used for drug delivery systems to release the drugs in a controlled manner (Borges et al., 2021). The reversible thermo-responsive property of poloxamer 407 hydrogel allows it to undergo gelation near body temperature (approximately $37^{\circ} \mathrm{C}$ ) and remain a continuous drug delivery device in vivo (Beard et al., 2021b). At lower temperatures, poloxamer 407 exists in the form of a solution, during which it can be loaded with therapeutics for later release from its gel state (Beard et al., 2021a). A previous study has demonstrated that poloxamer 407 hydrogel loaded with drugs can be successfully injected into porous scaffolds and achieve sustained release (Bai H. T. et al., 2020).

\section{The Release Profiles of the 3D-Printed Composite Scaffolds}

For a drug delivery system, sustained release is critical to achieving the intended therapeutic effect. The release profiles of the BMP-2/ OPG-loaded 3D-printed composite scaffolds indicated that the slow degradation of hydrogels allowed the BMP-2 and OPG to be delivered in a controlled manner. Figures 2A,B show that the kinetic release of BMP-2 and OPG was recorded in 20 days. Initially, there was a rapid release of drugs in the first 4 days, especially on day 1 . For BMP-2, the release rate was about $20.9 \% \pm 1.1 \%$ at day 1 . On day 4 , the percentage of the released drug was approximately $44.5 \% \pm 2.3 \%$. From day 4 to day 16 , the release rate slowed down; and on day 16, the total fraction of drug release was $69.6 \% \pm 1.3 \%$. After that, the release profile increased slowly. As for OPG, the release rate was about $23.1 \% \pm 2.3 \%$ on day 1 and approximately $49.9 \% \pm 2.7 \%$ on day 4 . Similar to BMP-2, the release rate slowed down from day 4 to day 16 , with a total fraction of drug release of $61.1 \% \pm 1.2 \%$ at day 16 . Because of protein denaturation and low initial drug concentration, after 20 days, the residual drug content was very low. The residual drug concentration was difficult to measure accurately; that is why only $70 \%$ of the drugs were detected. The release of drugs in the hydrogel was the result of drug diffusion and hydrogel degradation. At the early time point, the rapid drug release mainly resulted from the drug diffusion since there was no significant hydrogel degradation. Moreover, because of the porous structure of both hydrogels and scaffolds, there was a broad hydrogel-liquid interface (Colucci et al., 2021). Through the diffusion of drugs on the surface, the broad interface was initially mainly responsible for rapid release, which was acceptable in the clinic and consistent with drug delivery patterns in other studies (Katakam et al., 2019; Tundisi et al., 2021). Later, the degradation rate was influenced by the drug diffusion and hydrogel degradation and the interlink of chemical bonds and intermolecular hydrogen bonds between drugs and the poloxamer 407 (Borges et al., 2021). Thus, the sustained release of drugs was achieved (Solanki et al., 2019). Because the protein concentration is impossible to detect in vivo, in vitro experiments simulated the in vivo release pattern. In vitro, we studied the release curves of growth factors in composite scaffolds in PBS. PBS maintains a constant $\mathrm{pH}$, and the osmotic pressure and ion concentration are similar to those of the human body, and they simulate the effect of body fluid. Therefore, it is reasonable to believe that the growth factors in the composite scaffolds can obtain a similar release curve in vivo. This degradation mode will meet the clinical requirements, and the initial rapid release is conducive to the drug reaching a high concentration at the defect and playing a rapid therapeutic role while alleviating the disease. The slow release in the later stage is beneficial to maintaining the drug concentration (Santimetaneedol et al., 2019). These results indicate that the poloxamer 407 hydrogel was an excellent drug delivery system for sustained release in bone regeneration.

\section{The Biocompatibility of the 3D-Printed Composite Scaffolds}

In addition to sustained drug release, the biocompatibility of the $3 \mathrm{D}$-printed composite system is the basis for biological applications. To test the biocompatibility of the BMP-2/OPG-loaded 3D-printed composite scaffolds, a CCK- 8 assay was conducted to analyze its effect on cell proliferation. OP-BMSCs were seeded on 3D-printed composite scaffolds in different groups for 1, 4, and 7 days. Figure 2C indicates that cells proliferated gradually in each group within 7 days. There was no significant difference between the groups, but the drug-loaded scaffold groups showed an increased cell proliferation rate on day 7. Previous studies reported that BMP-2 and OPG were mainly responsible for cell differentiation, with little effect on cell proliferation (Yarygin et al., 2020; Wang et al., 2021; Zhang et al., 2021). In addition, the images of Calcein-AM/PI staining indicated that the OP-BMSCs had good 

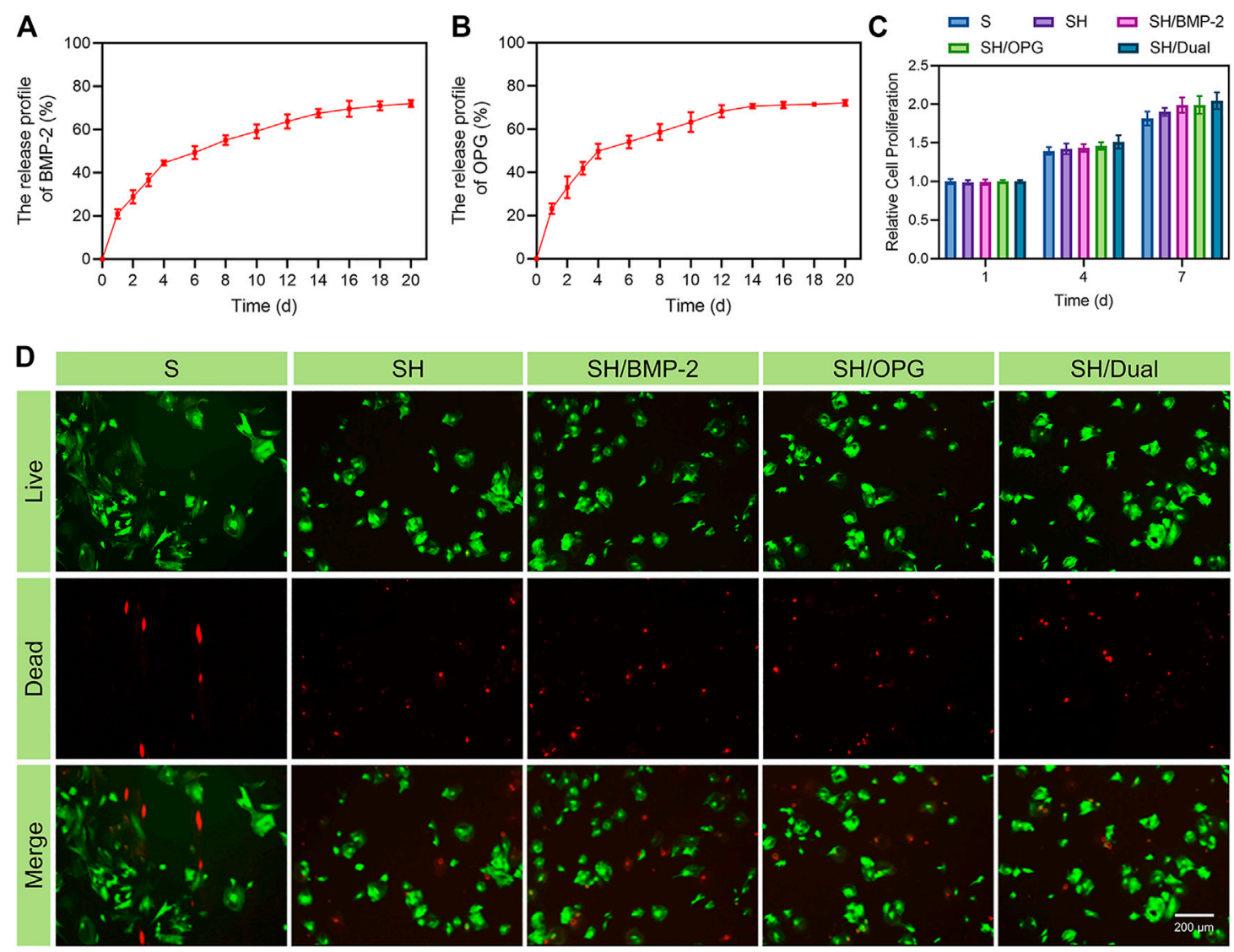

FIGURE 2 | The release profiles and biocompatibility of the 3D-printed composite scaffolds. (A) The release profile of BMP-2. (B) The release profile of OPG. (C) The cell proliferation of BMSCs in different groups. (D) Calcein-AM/PI staining of BMSCs at day 3 (green represents live cells, whereas red represents dead cells). BMP-2, bone morphogenetic protein-2; OPG, osteoprotegerin; BMSCs, bone marrow mesenchymal stem cells; PI, propidium iodide.

cell viability in all groups on day 3 (Figure 2D). These results indicated that the incorporated drugs, hydrogels, and scaffolds used in this study had no cytotoxicity, suggesting that the BMP-2/OPGloaded 3D-printed composite scaffolds had good biocompatibility and were suitable for in vivo applications.

\section{Osteogenic and Osteoclastic Differentiation on the 3D-Printed Composite Scaffolds}

In addition to cell proliferation, osteogenic differentiation of BMSCs is an important event for the initiation of bone regeneration. ARS attaining, which indicated the number of calcified deposits in BMSCs (Queiroz et al., 2021), was used to evaluate the osteogenic effects of the 3D-printed composite scaffolds on OP-BMSCs. Gross images revealed that calcified nodules were increased from 14 to 21 days in each group (Figure 3A), but there was no significant difference among the $\mathrm{S}, \mathrm{SH}$, and $\mathrm{SH} / \mathrm{OPG}$ groups at each time point. Moreover, OPG did not have significant effects on calcium deposition. As predicted, the BMP-2-loaded groups had significantly more calcified nodules than the $\mathrm{S}$ and $\mathrm{SH}$ groups at both time points (Figure 3B). The difference in nodules increased as time went on $(p<0.05$ at day 14 and $p<0.001$ at day 21$)$.
More importantly, we observed more calcium deposition in the $\mathrm{SH} /$ Dual group compared with the SH/BMP-2 group $(p<0.05)$. These results demonstrated that compared with BMP-2 alone, although OPG did not significantly affect osteogenesis, the cooperation of BMP-2 and OPG promoted mineralized matrix formation more significantly.

To further analyze the effect of the BMP-2/OPG-loaded 3Dprinted composite scaffolds on osteogenic differentiation and osteoclastic differentiation, the expression of related genes, including Runx-2, OPN, and RANKL, were evaluated via RTqPCR. Typically, Runx-2 is one of the transcription factors in the Runx family (Song et al., 2020). It is considered an earlystage marker that indicates the differentiation of osteoblasts (Zahid and Ghafoor, 2021). Figure 3C shows that on both days 14 and 21, the expression of Runx-2 was significantly higher in SH/BMP-2 than in the $\mathrm{S}$ and $\mathrm{SH}$ groups. More importantly, the expressions of Runx-2 in SH/Dual were significantly higher than in SH/BMP-2 in both time points. This means that the existence of OPG can more significantly improve bone regeneration compared with BMP-2 alone. OPN is a glycophosphoprotein in the extracellular matrix that plays an essential role during osteoblastic differentiation (Han et al., 2021). From Figure 3D, the activity of $O P N$ was significantly upregulated in BMP-2-loaded composite scaffolds on day 14 and 

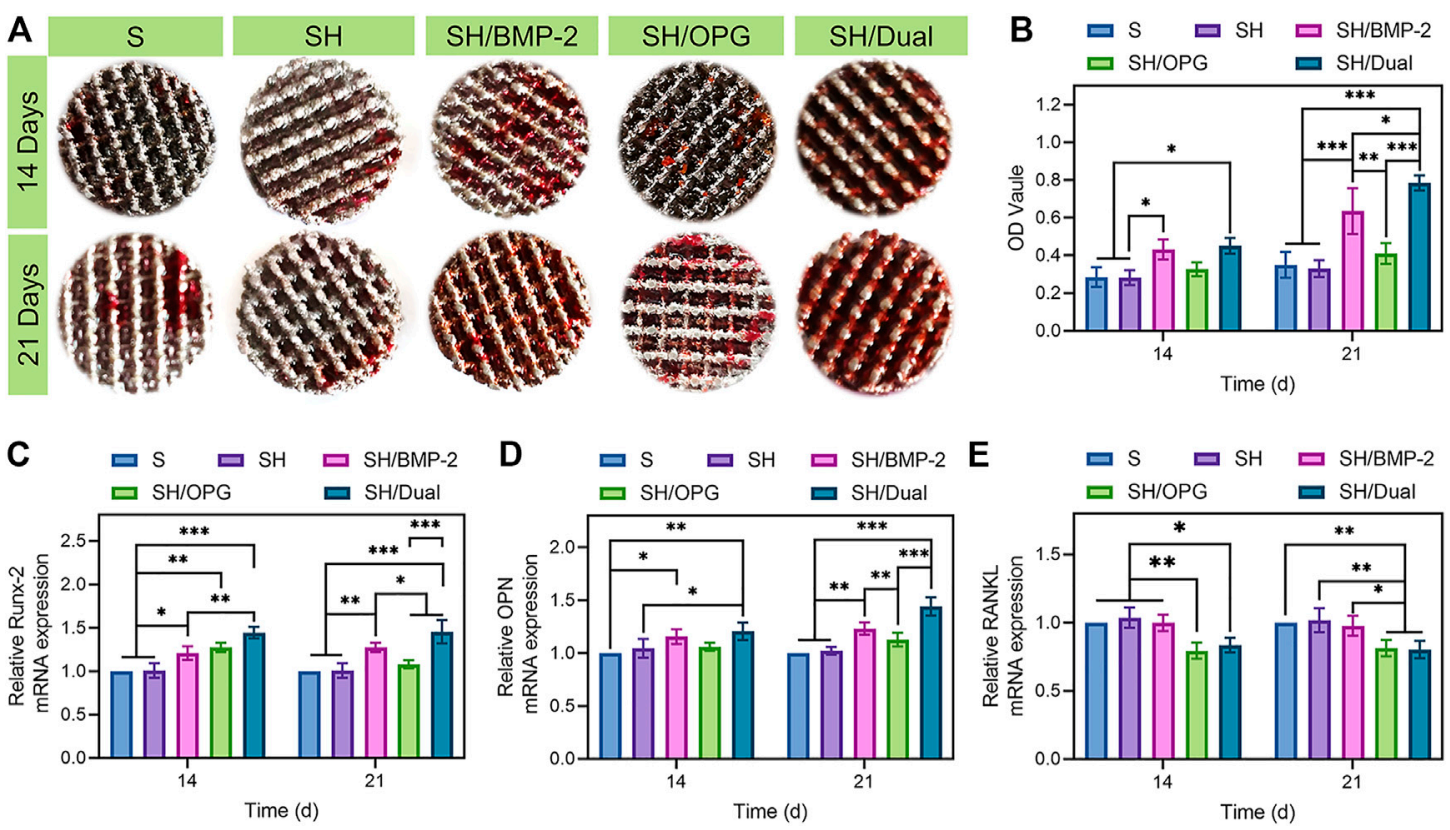

FIGURE 3 | The osteogenic and osteoclastic activity of BMSCs on different 3D-printed composite scaffolds. (A) The alizarin red staining of the 3D-printed composite scaffolds in different groups. (B) Quantitative analysis of the ARS results. (C-E) RT-qPCR analyses of the gene expressions of Runx-2, OPN, and RANKL. * indicates significant difference between groups, ${ }^{*} p<0.05,{ }^{\star \star} p<0.01$, and ${ }^{* \star *} p<0.001$. BMSCs, bone marrow mesenchymal stem cells.

day 21. However, though there was no significant difference between the SH/Dual and SH/BMP-2 groups, and the SH/Dual group showed higher expression of $O P N$ than did the other groups. Figures 3B-D clearly show a similar trend of osteogenesis between the SH/Dual group and the SH/BMP-2 group. At the early time point, they had a similar effect on bone regeneration, but as time went on, the expression levels of osteogenic differentiation markers tended to be higher in the SH/Dual group. This phenomenon may result from BMP-2 inducing the osteoblastic differentiation of BMSCs at the early time point. Lately, the existence of OPG inhibited the osteoclast-related differentiation, which maintains the osteogenic environment.

Under osteoporotic conditions, adipogenesis and osteoclastogenesis are enhanced while osteogenesis is inhibited (Cosman et al., 2014). For BMSCs (well-known precursor cells for adipocytes and osteoblasts), under osteoporotic conditions, adipogenesis and osteoclastogenesis overwhelm osteogenesis (Shen et al., 2018). The osteoclastogenesis process is achieved through RANKL/OPG/RANK pathway (Lai et al., 2020) and osteoclastogenic cytokines including interleukin-6 (IL-6), TNF- $\alpha$, and macrophage colony-stimulating factor (M-CSF) (Huang et al., 2020). RANKL, secreted by osteoblasts, can induce osteoclast function through binding to RANK on the surface of osteoclasts (Zhang et al., 2015). In experiments performed in vitro, the expression levels of RANKL in OP-BMSCs are often used to detect the effects of osteoclastogenesis (Bai H. T. et al., 2020). In this study, further evidence was offered by measuring the expression of osteoclast-related genes to make this point clearer. The RANKL gene can regulate the differentiation and function of osteoclasts by binding to RANK, which is located on the osteoclast membrane
(Cang et al., 2021). The activation of RANK promotes osteoclastic effects and increases bone resorption (Liu et al., 2021). Therefore, the lower expression level of RANKL reveals a lower level of osteoclastic differentiation. In Figure 3E, both the SH/OPG and SH/Dual groups showed reduced levels of RANKL expression than did the other three groups. All these statistical differences indicated that the cooperation of BMP-2 and OPG significantly improved osteogenic differentiation and inhibited osteoclastic activation. Therefore, it is predictable that the dual BMP-2/OPG-loaded 3Dprinted composite scaffolds will regulate the osteoporotic microenvironment to promote osteogenesis and inhibit osteolysis, thus promoting osseointegration after joint replacement.

\section{Validation of the Ovariectomy Rabbit Models}

All the female rabbits were kept alive, and no infection or other surgical complications occurred throughout the experiment. In osteoporotic animals, the balance between bone resorption and bone regeneration is broken. This means that enhanced bone resorption will cause decreased $\mathrm{BMD}$, which will increase the risk of fracture. As previously demonstrated, the OVX rabbits will have a deficiency of estrogen levels, and low estrogen concentration can inhibit osteoblast function and promote bone resorption (Permuy et al., 2019). In this way, the osteoporotic animals were established by OVX. To verify the establishment of the osteoporotic model, six rabbits were sacrificed 10 months after the OVX surgery, including three OVX and three sham rabbits. From Supplementary Figure S1, the H\&E staining of distal femur slices indicated that the trabecular structure in the OVX group was much 


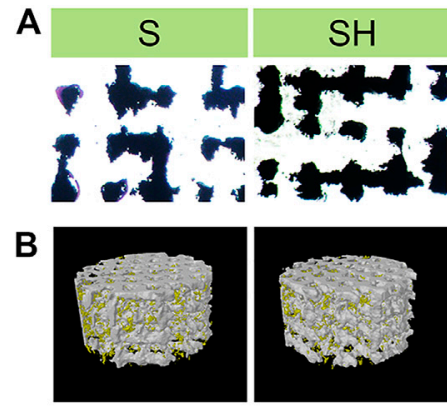

D

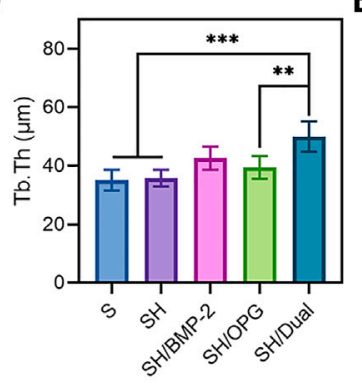

H

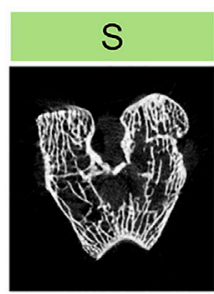

SH/BMP-2

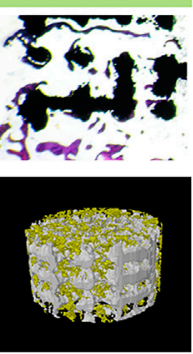

E
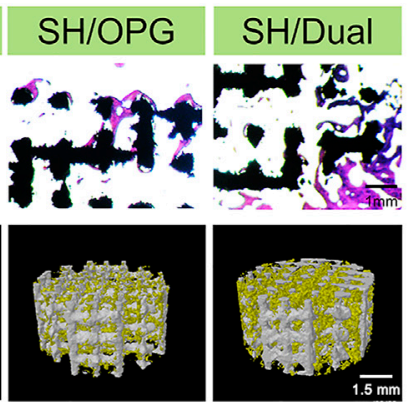

$\mathbf{F}$

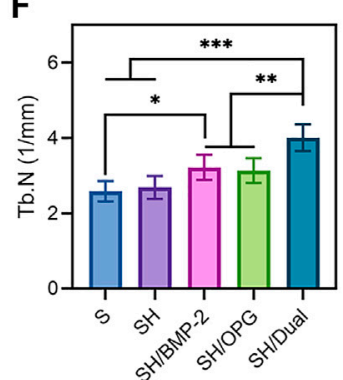

C

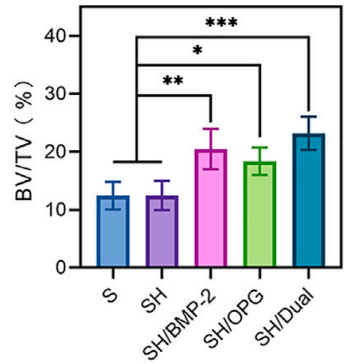

G

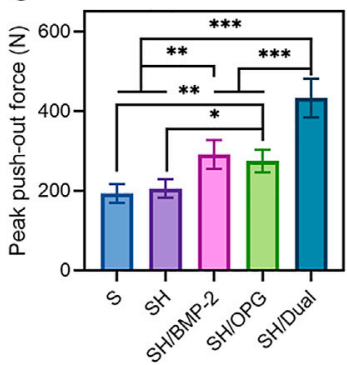

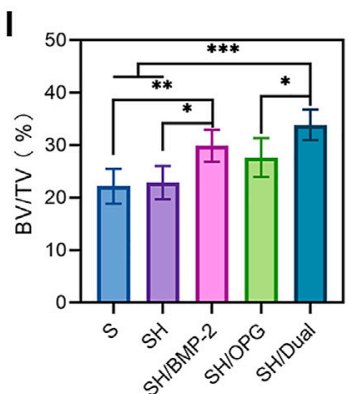

FIGURE 4 | Bone regeneration and osseointegration in the 3D-printed composite scaffolds. (A) Masson's trichrome staining of the regenerated bone tissue in and around the 3D-printed composite scaffolds. (B) 3D reconstruction images of different groups (the yellow substance represents the new bone tissue). (C-F) Micro-CT analyses of BV/TV, Tb.Th, Tb.Sp, and Tb.N in each group. (G) The analysis of the push-out test. (H) The CT images of the distal end of the implants in each group. (I) Quantitative analysis of the 2 -cm-long femur at the distal end in each group. ${ }^{*}$ indicates significant difference between groups, ${ }^{*} p<0.05$, ${ }^{* *} p<0.01$, and ${ }^{* \star *} p<$ 0.001. BV/TV, bone volume/tissue volume ratio; Tb.Th, trabecular thickness; Tb.Sp, trabecular separation; Tb.N, trabecular number.

thinner and looser than in the sham groups. In addition, the serum estrogen level in non-OVX rabbits was 3.2-fold higher than in OVX rabbits (Supplementary Figure S2). The statistical analyses also demonstrated that BV/TV (Supplementary Figure S3) and BMD (Supplementary Figure S4) in OVX rabbits were significantly decreased compared with those in the sham group $(p<0.05)$. In summary, all data collectively confirmed that the osteoporotic rabbits were successfully established 10 months after OVX surgery.

\section{Osseointegration of the 3D-Printed Composite Scaffolds in Osteoporosis}

After validating the osteoporotic animal models, the OVX rabbits were used to evaluate the osseointegration efficiency of BMP-2/ OPG-loaded 3D-printed composite scaffolds. The composite scaffolds were successfully implanted into the defects at the distal femur in the osteoporotic rabbits. Masson's trichrome staining was performed 3 months after implantation to observe the bone ingrowth and bone interface bonding. From Figure 4A, it was found that there was more bone ingrowth in the BMP-2- and OPG-loaded groups, while less was observed in the $\mathrm{S}$ and $\mathrm{SH}$ groups. In addition, much more bone regeneration was revealed in the SH/Dual group compared with the SH/BMP-2 and SH/OPG groups. Moreover, Micro-CT was employed to evaluate new bone formation in the implanted site. The $3 \mathrm{D}$ reconstruction images of the porous scaffolds are shown in Figure 4B, where the yellow part represents the regenerated bone tissue and the white part indicates the porous scaffolds. It was clear to see more bone tissue on the surface and in the pores in the SH/Dual group and moderate tissue in the SH/BMP-2 and SH/OPG groups. However, limited bone formation was found in the $\mathrm{S}$ and $\mathrm{SH}$ groups. Quantitative morphological results of Micro-CT were further analyzed and depicted in Figures 4C-F. The BV/TV values of $\mathrm{S}, \mathrm{SH}, \mathrm{SH} /$ BMP-2, SH/OPG, and SH/Dual were $12.41 \% \pm 0.93 \%, 12.47 \% \pm$ $2.50 \%, 20.45 \% \pm 3.84 \%, 18.39 \% \pm 2.63 \%$, and $23.18 \% \pm 2.84 \%$, respectively. Furthermore, the results of Tb.Th, Tb.Sp, and Tb.N 

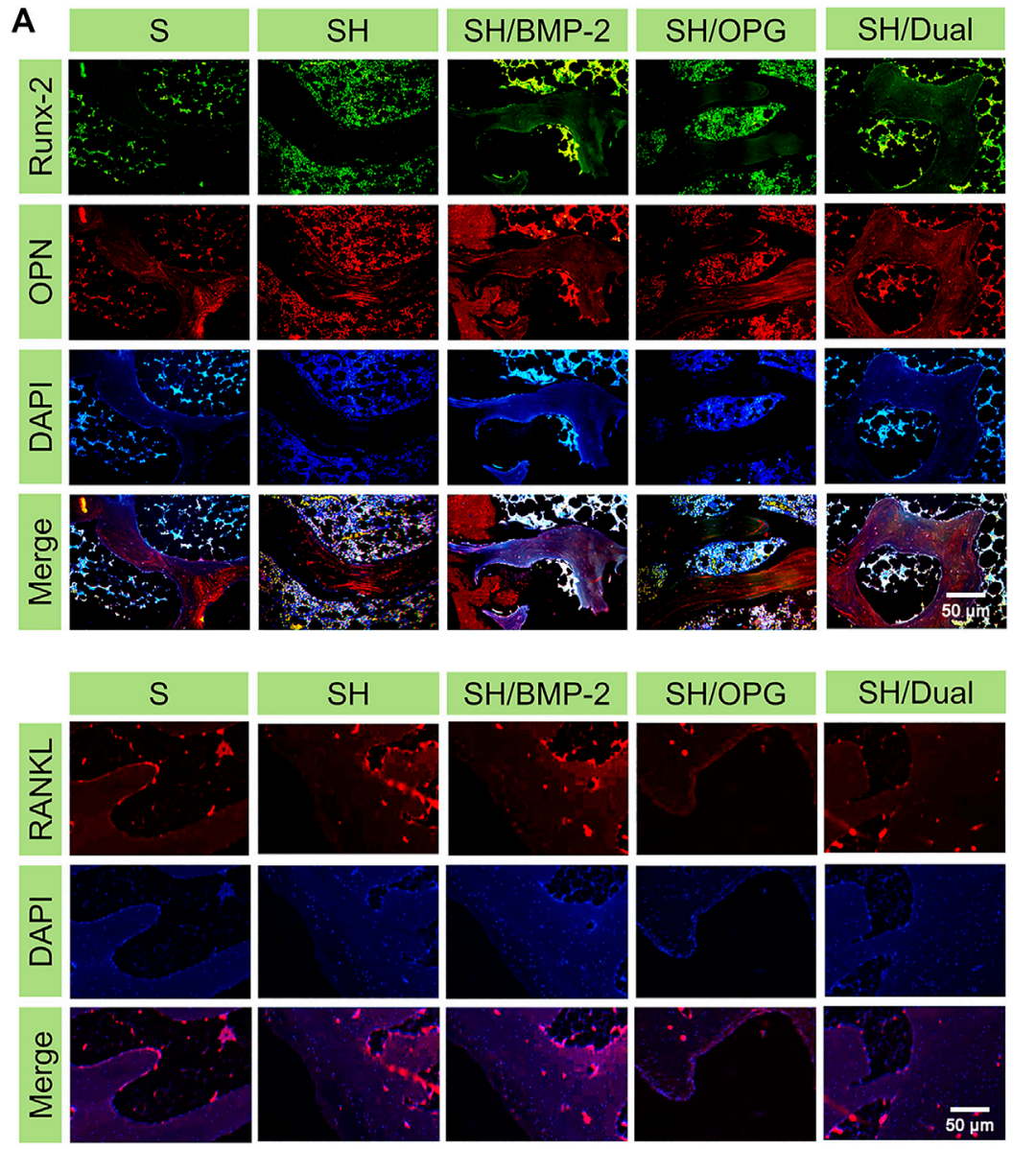
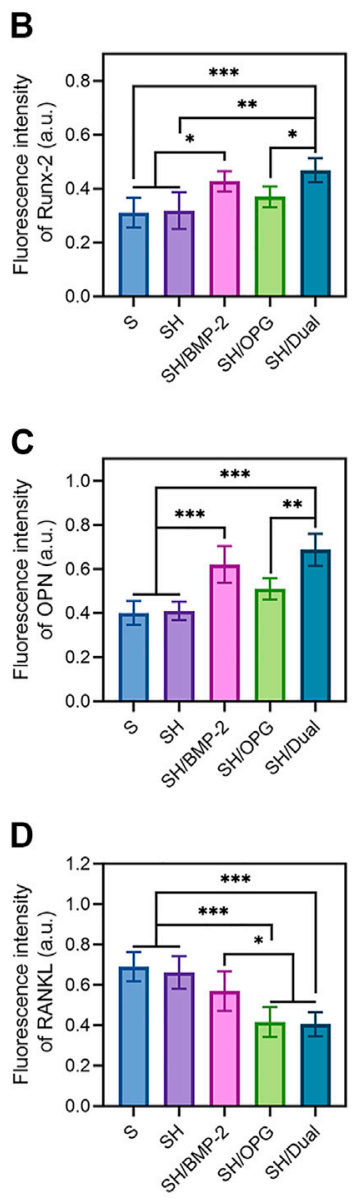

FIGURE 5 | Immunocytochemical staining of the bone interface in each group. (A) The immunocytochemical images of osteogenic- and osteoclastic-related proteins (Runx-2, OPN, and RANKL) in each group. (B-D) The quantitative analyses of Runx-2, OPN, and RANKL expression. * indicates significant difference between groups, ${ }^{\star} p<0.05,{ }^{\star *} p<0.01$, and ${ }^{\star \star *} p<0.001$

also indicated that BMP-2 and OPG could significantly enhance bone mass and quality in the defects. Taken together, the histological and micro-CT results revealed that the BMP-2/OPGloaded 3D-printed composite scaffolds could promote bone regeneration and bone ingrowth after porous prosthesis implantation.

The probability of prosthesis displacement and loosening and periprosthetic fracture can be predicted by evaluating the osseointegration strength of the interface and the surrounding bone mass (Kang et al., 2018; Cai et al., 2020). The push-out test was conducted to evaluate the osseointegration strength of the interface between the implants and surrounding bone (Figure 4G). The maximum push-out force values of the SH/ Dual group were 1.5-fold and 1.6-fold higher than those of the SH/BMP-2 and SH/OPG groups $(p<0.001)$, and 2.2-fold and 2.1fold higher than those of the $\mathrm{S}$ and $\mathrm{SH}$ groups $(p<0.001)$, respectively. It is well known that some severe complications, including prosthesis displacement and loosening, are because of the weak bone integration of the prosthesis and surrounding bone. Therefore, the dual existence of BMP-2 and OPG can significantly reduce the risk of implant loosening and displacement. In addition, the occurrence of periprosthetic fracture is often related to the bone mass around the prosthesis after joint replacement. In this study, we performed a micro-CT scan and quantitative analysis. The CT images of the distal end of the implants indicated that the $\mathrm{SH} /$ Dual group had the densest trabecular bone structure among the groups (Figure $4 \mathbf{H}$ ). Quantitative analysis of the 2-cm-long femur at the distal end of the femurs where the implants were located revealed that the BV/TV values of S, SH, SH/BMP-2, SH/OPG, and SH/Dual were $22.21 \% \pm 2.98 \%, 22.91 \% \pm 2.83 \%, 29.89 \% \pm 2.71 \%, 27.66 \% \pm$ $3.30 \%$, and $33.88 \pm 2.61 \%$, respectively (Figure $4 \mathrm{I}$ ). These results indicated that BMP-2/OPG-loaded 3D-printed composite scaffolds could improve the bone mass around the implants, which can be expected to reduce the occurrence of periprosthetic fractures.

\section{Bone Formation and Resorption Markers on the Interface}

To evaluate the expression of the bone formation and bone resorption-related markers, Runx-2, OPN, and RANKL were labeled via immunofluorescence staining at the bone interface after scaffolds were pushed out (Figure 5A). Figures 5B,C show 
that the expressions of Runx-2 and OPN were much higher in the $\mathrm{SH} / \mathrm{BMP}-2$ and SH/Dual groups than in the S, SH, and SH/OPG groups. As expected, the osteogenic-related proteins were higher in the SH/Dual groups compared with BMP-2 alone by using fluorescence intensity analyses. It is well known that the level of Runx-2 and OPN reflects a bone-regenerating environment (James et al., 2016; Wang et al., 2016). The fluorescence intensity analysis demonstrated that the existence of OPG reduced RANKL expression levels, thus inhibiting osteoclast activity. The cooperation of BMP-2 and OPG decreased RANKL to a higher degree compared with OPG loading alone (Figure 5D). This result may be because the cooperation of BMP-2 increased the regeneration of osteoblasts (Zheng et al., 2021). The activation of osteoblasts improved the osteogenic microenvironment in osteoporotic defects, and as a result, the osteoclast function was inhibited (Kim et al., 2021). The synergistic release of BMP-2 and OPG enhanced the osteogenic activity, inhibited the osteoclastic activity around the osteoporotic bone interface, and finally promoted the osseointegration of the 3Dprinted composite scaffolds.

\section{CONCLUSION}

To address the challenges of poor osseointegration in patients with osteoporosis after joint replacement, we developed a novel 3Dprinted bioactive system that allowed for the sustained release of growth factors. In this study, 3D-printed Ti6Al4V porous scaffolds were filled with BMP-2- and OPG-loaded hydrogels. The superior efficacy of this therapy was systematically proved by its biological functions of maintaining osteoporosis-derived BMSC proliferation, viability, and differentiation in vitro and significantly improving bone generation and osseointegration. Therefore, this provides a new strategy for reducing postoperative complications and improving the outcome of joint replacement in patients with osteoporosis.

\section{DATA AVAILABILITY STATEMENT}

The original contributions presented in the study are included in the article/Supplementary Material, further inquiries can be directed to the corresponding authors.

\section{REFERENCES}

Abate, K. M., Nazir, A., and Jeng, J. Y. (2021). Design, Optimization, and Selective Laser Melting of Vin Tiles Cellular Structure-Based Hip Implant. Int. J. Adv. Manufacturing Techn. 112 (7-8), 2037-2050. doi:10.1007/s00170020-06323-5

Baek, H. J., Kim, I. H., Yun, P. Y., and Kim, Y. K. (2021). Prognosis of Single Tooth Implants Following Alveolar ridge Preservation with Two Recombinant Human Bone Morphogenetic Protein-2 Delivery Systems. Bmc Oral Health 21 (1), 201. doi:10.1186/s12903-021-01565-5

Bai, H. T., Cui, Y. T., Wang, C. Y., Wang, Z. H., Luo, W. B., Liu, Y. Z., et al. (2020b). 3D Printed Porous Biomimetic Composition Sustained Release Zoledronate to Promote Osteointegration of Osteoporotic Defects. Mater. Des. 189, 108513. doi:10.1016/j.matdes.2020.108513

Bai, H., Zhao, Y., Wang, C., Wang, Z., Wang, J., Liu, H., et al. (2020a). Enhanced Osseointegration of Three-Dimensional Supramolecular Bioactive Interface

\section{ETHICS STATEMENT}

The animal study was reviewed and approved by the Animal Care and Use Ethics Committee of Jilin University.

\section{AUTHOR CONTRIBUTIONS}

XW and ZhL: conceptualization, investigation, data curation, and writing-original draft. ZW: formal analysis. HL: project administration and funding acquisition. $\mathrm{YC}$ and $\mathrm{YL}$ : software. MR and HZ: validation. ZuL: methodology, supervision, and writing-review and editing. MW and JW: resources, supervision, and writing-review and editing. All authors contributed to the article and approved it for publication.

\section{FUNDING}

This work was supported by the National Key Technologies Research and Development (R\&D) Program of China (grant number 2018YFB1105100), the National Natural Science Foundation of China (grant number 82001971), the Jilin Scientific and Technological Development Program (grant numbers 20200403088SF, 20200802008GH, 20200404202YY, 20200404140YY, 20190304123YY, 20200404190YY, 20180201041SF, and 20180623050TC), the Program of Jilin Provincial Health Department (grant numbers 2019SCZT001, 2019SCZT014, 2019SRCJ001, and 2017F007), Youth Science and Technology Training Program of Jilin Provincial Health Department (grant number 2020Q018), and the Youth Talents Promotion Project of Jilin Province (grant number 192004).

\section{SUPPLEMENTARY MATERIAL}

The Supplementary Material for this article can be found online at: https://www.frontiersin.org/articles/10.3389/fbioe.2021.754205/ full\#supplementary-material

through Osteoporotic Microenvironment Regulation. Theranostics 10 (11), 4779-4794. doi:10.7150/thno.43736

Beard, M. C., Cobb, L. H., Grant, C. S., Varadarajan, A., Henry, T., Swanson, E. A., et al. (2021a). Autoclaving of Poloxamer 407 Hydrogel and its Use as a Drug Delivery Vehicle. J. Biomed. Mater. Res. B-Applied Biomater. 109 (3), 338-347. doi:10.1002/jbm.b.34703

Beard, M. C., Cobb, L. H., Grant, C. S., Varadarajan, A., Henry, T., Swanson, E. A., et al. (2021b). Autoclaving of Poloxamer 407 Hydrogel and its Use as a Drug Delivery Vehicle. J. Biomed. Mater. Res. B Appl. Biomater. 109 (3), 338-347. doi:10.1002/jbm.b.34703

Borges, R., Kai, K. C., Lima, C. A., Zezell, D. M., de Araujo, D. R., and Marchi, J. (2021). Bioactive Glass/poloxamer 407 Hydrogel Composite as a Drug Delivery System: The Interplay between Glass Dissolution and Drug Release Kinetics. Colloids Surf. B Biointerfaces 206, 111934. doi:10.1016/j.colsurfb.2021.111934

Cai, J., Shao, X., Yang, Q., Yang, Y., Yan, Z., Luo, E., et al. (2020). Pulsed Electromagnetic fields Modify the Adverse Effects of Glucocorticoids on Bone Architecture, Bone Strength and Porous Implant Osseointegration by 
Rescuing Bone-Anabolic Actions. Bone 133, 115266. doi:10.1016/ j.bone.2020.115266

Cang, D. W., Zou, G. Y., Yang, C., Shen, X. F., Li, F., Wu, Y., et al. (2021). Dencichine Prevents Ovariectomy-Induced Bone Loss and Inhibits Osteoclastogenesis by Inhibiting RANKL-Associated NF-Kappa B and MAPK Signaling Pathways. J. Pharmacol. Sci. 146 (4), 206-215. doi:10.1016/ j.jphs.2021.04.004

Chao, Y. L., Wang, T. M., Chang, H. H., and Lin, L. D. (2021). Effects of Low-Dose rhBMP-2 on Peri-Implant ridge Augmentation in a Canine Model. J. Clin. Periodontol. 48 (5), 734-744. doi:10.1111/jcpe.13440

Chen, Z., Yan, X., Yin, S., Liu, L., Liu, X., Zhao, G., et al. (2020). Influence of the Pore Size and Porosity of Selective Laser Melted Ti6Al4V ELI Porous Scaffold on Cell Proliferation, Osteogenesis and Bone Ingrowth. Mater. Sci. Eng. C, Mater. Biol. Appl. 106, 110289. doi:10.1016/j.msec.2019.110289

Colucci, F., Mancini, V., Mattu, C., and Boffito, M. (2021). Designing Multifunctional Devices for Regenerative Pharmacology Based on 3D Scaffolds, Drug-Loaded Nanoparticles, and Thermosensitive Hydrogels: A Proof-Of-Concept Study. Pharmaceutics 13 (4), 464. doi:10.3390/ pharmaceutics13040464

Compston, J. E., McClung, M. R., and Leslie, W. D. (2019). Osteoporos. Lancet 393 (10169), 364-376. doi:10.1016/S0140-6736(18)32112-3

Cosman, F., de Beur, S. J., LeBoff, M. S., Lewiecki, E. M., Tanner, B., Randall, S., et al. (2014). Clinician's Guide to Prevention and Treatment of Osteoporosis. Osteoporos. Int. 25 (10), 2359-2381. doi:10.1007/s00198-014-2794-2

Ferrari, S. L. (2018). Prevention of Fractures in Patients with Osteoporosis. Lancet 391 (10117), 184-186. doi:10.1016/S0140-6736(17)32167-0

Fitzpatrick, V., Fourel, L., Destaing, O., Gilde, F., Albiges-Rizo, C., Picart, C., et al. (2017). Signal Mingle: Micropatterns of BMP-2 and Fibronectin on Soft Biopolymeric Films Regulate Myoblast Shape and SMAD Signaling. Sci. Rep. 7, 41479. doi:10.1038/srep41479

Frank, M., Grabos, A., Reisinger, A. G., Burr, D. B., Pahr, D. H., Allen, M. R., et al. (2021). Effects of Anti-resorptive Treatment on the Material Properties of Individual Canine Trabeculae in Cyclic Tensile Tests. Bone 150, 115995. doi:10.1016/j.bone.2021.115995

Garcia-Garcia, P., Reyes, R., Segredo-Morales, E., Perez-Herrero, E., Delgado, A., and Evora, C. (2019). PLGA-BMP-2 and PLA-17beta-Estradiol Microspheres Reinforcing a Composite Hydrogel for Bone Regeneration in Osteoporosis. Pharmaceutics 11 (12), 648. doi:10.3390/pharmaceutics 11120648

Guo, W., Li, H., Lou, Y., Zhang, Y., Wang, J., Qian, M., et al. (2021). Tyloxapol Inhibits RANKL-Stimulated Osteoclastogenesis and Ovariectomized-Induced Bone Loss by Restraining NF-kappaB and MAPK Activation. J. Orthop. Translat 28, 148-158. doi:10.1016/j.jot.2021.01.005

Han, X. Y., Sun, M. J., Chen, B., Saiding, Q., Zhang, J. Y., Song, H. L., et al. (2021). Lotus Seedpod-Inspired Internal Vascularized 3D Printed Scaffold for Bone Tissue Repair. Bioactive Mater. 6 (6), 1639-1652. doi:10.1016/ j.bioactmat.2020.11.019

Hauser, B., Zhao, S. Z., Visconti, M. R., Riches, P. L., Fraser, W. D., Piec, I., et al. (2017). Autoantibodies to Osteoprotegerin Are Associated with Low Hip Bone Mineral Density and History of Fractures in Axial Spondyloarthritis: A CrossSectional Observational Study. Calcified Tissue Int. 101 (4), 375-383. doi:10.1007/s00223-017-0291-2

Huang, L. Z., Cai, B. Y., Huang, Y., Wang, J. C., Zhu, C., Shi, K., et al. (2021). Comparative Study on 3D Printed Ti6Al4V Scaffolds with Surface Modifications Using Hydrothermal Treatment and Microarc Oxidation to Enhance Osteogenic Activity. Acs Omega 6 (2), 1465-1476. doi:10.1021/ acsomega.0c05191

Huang, Y., Yin, Y., Gu, Y., Gu, Q., Yang, H., Zhou, Z., et al. (2020). Characterization and Immunogenicity of Bone Marrow-Derived Mesenchymal Stem Cells under Osteoporotic Conditions. Sci. China Life Sci. 63 (3), 429-442. doi:10.1007/ s11427-019-1555-9

James, A. W., LaChaud, G., Shen, J., Asatrian, G., Nguyen, V., Zhang, X., et al. (2016). A Review of the Clinical Side Effects of Bone Morphogenetic Protein-2. Tissue Eng. Part. B Rev. 22 (4), 284-297. doi:10.1089/ ten.TEB.2015.0357

Jia, X., Yang, M., Hu, W., and Cai, S. (2021). Overexpression of miRNA-22-3p Attenuates Osteoporosis by Targeting MAPK14. Exp. Ther. Med. 22 (1), 692. doi:10.3892/etm.2021.10124
Kang, H. G., Jeong, Y. S., Huh, Y. H., Park, C. J., and Cho, L. R. (2018). Impact of Surface Chemistry Modifications on Speed and Strength of Osseointegration. Int. J. Oral Maxillofac. Implants 33 (4), 780-787. doi:10.11607/jomi.5871

Katakam, P., Banapuram, S. R., and Adiki, S. K. (2019). Formulation, Evaluation and Cytotoxic Potential of Metronidazole Loaded Poloxamer 407 Hydrogel in Scc-29 Cell Lines. Int. J. Pharm. Sci. Res. 10 (3), 1354-1359. doi:10.13040/ Ijpsr.0975-8232.10(3).1354-59

Kim, K. J., Lee, J., Wang, W., Lee, Y., Oh, E., Park, K. H., et al. (2021). Austalide K from the Fungus Penicillium rudallense Prevents LPS-Induced Bone Loss in Mice by Inhibiting Osteoclast Differentiation and Promoting Osteoblast Differentiation. Int. J. Mol. Sci. 22 (11), 5493. doi:10.3390/ijms22115493

Lai, K., Xi, Y., Du, X., Jiang, Z., Li, Y., Huang, T., et al. (2020). Activation of Nell-1 in BMSC Sheet Promotes Implant Osseointegration through Regulating Runx2/ Osterix Axis. Front Cel Dev Biol 8, 868. doi:10.3389/fcell.2020.00868

Li, J., Wang, K. T., Bai, X. W., Wang, Q., Lv, N. Y., and Li, Z. L. (2021). Enhanced Regeneration of Bone Defects Using Sintered Porous Ti6Al4V Scaffolds Incorporated with Mesenchymal Stem Cells and Platelet-Rich Plasma. Rsc Adv. 11 (9), 5128-5138. doi:10.1039/d0ra10215f

Liu, Y., Wang, L., Yang, Y., and Xiong, J. (2021). Silencing Hoxa2 Reverses Dexamethasone-Induced Dysfunction of MC3T3-E1 Osteoblasts and Osteoporosis in Rats. Adv. Clin. Exp. Med. 30 (5), 525-534. doi:10.17219/ acem/133495

Monotti, I. C., Preston, C. A., and Kidd, S. W. (2020). Treatment Outcomes for Periprosthetic Femoral Fractures in Cementless Press-Fit Total Hip Replacement. Vet. Comp. Orthop. Traumatol. 33 (5), 370-376. doi:10.1055/ s-0040-1709486

Permuy, M., Lopez-Pena, M., Munoz, F., and Gonzalez-Cantalapiedra, A. (2019). Rabbit as Model for Osteoporosis Research. J. Bone Mineral Metab. 37 (4), 573-583. doi:10.1007/s00774-019-01007-x

Qiao, S. C., Sheng, Q. B., Li, Z. H., Wu, D. L., Zhu, Y., Lai, H. C., et al. (2020). 3Dprinted Ti6Al4V Scaffolds Coated with Freeze-Dried Platelet-Rich Plasma as Bioactive Interface for Enhancing Osseointegration in Osteoporosis. Mater. Des. 194, 108825. doi:10.1016/j.matdes.2020.108825

Queiroz, M. B., Torres, F. F. E., Rodrigues, E. M., Viola, K. S., Bosso-Martelo, R., Chavez-Andrade, G. M., et al. (2021). Physicochemical, Biological, and Antibacterial Evaluation of Tricalcium Silicate-Based Reparative Cements with Different Radiopacifiers. Dent Mater. 37 (2), 311-320. doi:10.1016/ j.dental.2020.11.014

Quinzi, D. A., Childs, S., Lipof, J. S., Soin, S. P., and Ricciardi, B. F. (2020). The Treatment of Periprosthetic Distal Femoral Fractures after Total Knee Replacement: A Critical Analysis Review. JBJS Rev. 8 (9), e2000003. doi:10.2106/JBJS.RVW.20.00003

Santimetaneedol, A., Wang, Z., Arteaga, D. N., Aksit, A., Prevoteau, C., Yu, M., et al. (2019). Small Molecule Delivery across a Perforated Artificial Membrane by Thermoreversible Hydrogel Poloxamer 407. Colloids Surf. B-Biointerfaces 182, 110300. doi:10.1016/j.colsurfb.2019.06.030

Segredo-Morales, E., Garcia-Garcia, P., Reyes, R., Perez-Herrero, E., Delgado, A., and Evora, C. (2018). Bone Regeneration in Osteoporosis by Delivery BMP-2 and PRGF from Tetronic-Alginate Composite Thermogel. Int. J. Pharm. 543 (12), 160-168. doi:10.1016/j.ijpharm.2018.03.034

Shen, G., Ren, H., Qiu, T., Zhang, Z., Zhao, W., Yu, X., et al. (2018). Mammalian Target of Rapamycin as a Therapeutic Target in Osteoporosis. J. Cel Physiol 233 (5), 3929-3944. doi:10.1002/jcp.26161

Solanki, N. G., Lam, K., Tahsin, M., Gumaste, S. G., Shah, A. V., and Serajuddin, A. T. M. (2019). Effects of Surfactants on Itraconazole-HPMCAS Solid Dispersion Prepared by Hot-Melt Extrusion I: Miscibility and Drug Release. J. Pharm. Sci. 108 (4), 1453-1465. doi:10.1016/j.xphs.2018.10.058

Song, J., Liu, L., Lv, L., Hu, S., Tariq, A., Wang, W., et al. (2020). Fluid Shear Stress Induces Runx-2 Expression via Upregulation of PIEZO1 in MC3T3-E1 Cells. Cell Biol Int 44 (7), 1491-1502. doi:10.1002/cbin.11344

Toepfer, E. T., Rott, J., Bartosova, M., Kolevica, A., Machuca-Gayet, I., Heuser, A., et al. (2021). Calcium Isotope Fractionation by Osteoblasts and Osteoclasts, across Endothelial and Epithelial Cell Barriers and with Binding to Proteins. Am. J. Physiol. Regul. Integr. Comp. Physiol. 321 (1), R29-R40. doi:10.1152/ ajpregu.00334.2020

Tu, P., Duan, P., Zhang, R. S., Xu, D. B., Wang, Y., Wu, H. P., et al. (2015). Polymorphisms in Genes in the RANKL/RANK/OPG Pathway Are Associated 
with Bone mineral Density at Different Skeletal Sites in post-menopausal Women. Osteoporos. Int. 26 (1), 179-185. doi:10.1007/s00198-014-2854-7

Tundisi, L. L., Yang, R., Borelli, L. P. P., Alves, T., Mehta, M., Chaud, M. V., et al. (2021). Enhancement of the Mechanical and Drug-Releasing Properties of Poloxamer 407 Hydrogels with Casein. Pharm. Res. 38 (3), 515-522. doi:10.1007/s11095-021-03017-9

Wang, Y. G., Qu, X. H., Yang, Y., Han, X. G., Wang, L., Qiao, H., et al. (2016). AMPK Promotes Osteogenesis and Inhibits Adipogenesis through AMPK-GfilOPN axis. Cell Signal. 28 (9), 1270-1282. doi:10.1016/j.cellsig.2016.06.004

Wang, Z., Bao, H. W., and Xu, Y. J. (2020). Cnidium Lactone Prevents Bone Loss in an Ovariectomized Rat Model through the Estrogen-alpha/BMP-2/Smad Signaling Pathway. J. Gene Med. 22 (8), e3198. doi:10.1002/jgm.3198

Wang, Z., Zhou, Y., Dai, Z., Chen, X., Li, C., Lin, Z., et al. (2021). A Novel Long Noncoding RNA, Lnc-OAD, Is Required for Bone Morphogenetic Protein 2(BMP-2-) Induced Osteoblast Differentiation. Biomed. Res. Int. 2021, 6697749. doi:10.1155/2021/6697749

Yang, H. M., Won, Y. H., Yoon, H. Y., Kim, C. H., Goo, Y. T., Chang, I. H., et al. (2020). Screening of Polymer Additives in Poloxamer 407 Hydrogel Formulations for Intravesical Instillation: Evaluation of Mechanical Properties, Gel-Forming Capacity, and Drug Release. Polymer-Korea 44 (6), 817-826. doi:10.7317/pk.2020.44.6.817

Yarygin, N. V., Parshikov, M. V., Prosvirin, A. A., Gur'ev, V. V., Govorov, M. V., Bosykh, V. G., et al. (2020). Effect of Morphogenetic Protein BMP-2 on X-Ray Density of Bone Defect in the Experiment. Bull. Exp. Biol. Med. 168 (4), 574-577. doi:10.1007/s10517-020-04755-3

Zahid, T., and Ghafoor, S. (2021). Molecular Events in the Clinicopathological Diagnosis of Alveolar Osteitis. J. Pakistan Med. Assoc. 71 (2), 508-513. doi:10.47391/Jpma.491

Zhang, P., Zhou, S., Chen, Z., Tian, Y., Wang, Q., Li, H., et al. (2021). TNF Receptor: Fc Fusion Protein Downregulates RANKL/OPG Ratio by Inhibiting CXCL16/CXCR6 in Active Ankylosing Spondylitis. Curr. Pharm. Biotechnol. 22 (2), 305-316. doi:10.2174/1389201021666200302104418

Zhang, Y., Ma, C., Liu, X., Wu, Z., Yan, P., Ma, N., et al. (2015). Epigenetic Landscape in PPARgamma2 in the Enhancement of Adipogenesis of Mouse
Osteoporotic Bone Marrow Stromal Cell. Biochim. Biophys. Acta 1852 (11), 2504-2516. doi:10.1016/j.bbadis.2015.08.020

Zhao, Y., Li, Z., Jiang, Y., Liu, H., Feng, Y., Wang, Z., et al. (2020). Bioinspired mineral Hydrogels as Nanocomposite Scaffolds for the Promotion of Osteogenic Marker Expression and the Induction of Bone Regeneration in Osteoporosis. Acta Biomater. 113, 614-626. doi:10.1016/ j.actbio.2020.06.024

Zheng, A., Wang, X., Wang, J., Xin, X. Z., Yu, Y. M., Liu, Y., et al. (2021). Synergistic Effect between 2-N,6-O-Sulfonated Chitosan and Bone Morphogenetic Protein-2. Carbohydr. Polym. 263, 11788. doi:10.1016/ j.carbpol.2021.117888

Zhu, K., Zhang, J., Zhang, C., Zhao, Z., Gao, J., Li, X., et al. (2021). Therapeutic Efficacy of Zoledronic Acid Combined with Calcitriol in Elderly Patients Receiving Total Hip Arthroplasty or Hemiarthroplasty for Osteoporotic Femoral Neck Fracture. Osteoporos. Int. 32 (3), 559-564. doi:10.1007/ s00198-020-05637-0

Conflict of Interest: The authors declare that the research was conducted in the absence of any commercial or financial relationships that could be construed as a potential conflict of interest.

Publisher's Note: All claims expressed in this article are solely those of the authors and do not necessarily represent those of their affiliated organizations, or those of the publisher, the editors, and the reviewers. Any product that may be evaluated in this article or claim that may be made by its manufacturer is not guaranteed or endorsed by the publisher.

Copyright $\odot 2021$ Wang, Li, Wang, Liu, Cui, Liu, Ren, Zhan, Li, Wu and Wang. This is an open-access article distributed under the terms of the Creative Commons Attribution License (CC BY). The use, distribution or reproduction in other forums is permitted, provided the original author(s) and the copyright owner(s) are credited and that the original publication in this journal is cited, in accordance with accepted academic practice. No use, distribution or reproduction is permitted which does not comply with these terms. 